\title{
Pulmonary fibrosis associated with TINF2 gene mutation: is somatic reversion required?
}

\begin{abstract}
To the Editor:
We read with great interest the case reported by FUKUHARA et al. [1] of a 43-year-old female patient with dyskeratosis congenita, pulmonary fibrosis and heterozygous mutation in TINF2 (telomerase repeat binding factor 1-interacting nuclear factor 2). TIN2, the TINF2 gene product, TERT (telomere reverse transcriptase) and TERC (telomerase RNA component) participate in the regulation of telomere elongation, in which mutations have been previously found to be associated with familial pulmonary fibrosis in adults [2]. Indeed mutations of SFTPC, coding for surfactant protein C, were initially described in children before being described in adults as old as 72 years of age who presented with familial pulmonary fibrosis [3].
\end{abstract}

However, we were surprised that a TINF2 mutation could be evidenced in an adult of that age. As highlighted by FUKUHARA et al. [1], patients with the TINF2 mutation present with severe haematological symptoms before 10 years of age [4]. As mentioned by FukUHARA et al. [1], the identified mutation is probably not hypomorphic because it is a frame-shift deletion located in the mutational "hot spot" described previously. Furthermore, the patient presented with very short telomeres. The TINF2 mutation was probably inherited from her father because he had abnormal skin pigmentation and aplastic anaemia [1].

Re-analysis of the gene mutation sequencing could provide new hypotheses for this late disease onset. Indeed, the electrophoregram depicted in figure $1 \mathrm{~b}$ in the study by FUKUHARA et al. [1] probably comes from a PCR product sub-cloned into an expression vector [5], and does not ensure that the deletion is at the heterozygous status usually seen in our patients (fig. 1). The patient may have experienced a somatic reversion leading to a partial loss of the germline mutation in peripheral blood cells (used for sequencing analysis). Indeed, cells would take advantage of a somatic reversion to become normal, particularly in the blood which is a highly regenerating system. This situation could explain the "milder" phenotype in this patient as compared with "classical" patients with TINF2 mutation. Of note, somatic reversion has been previously reported with TERC mutations [6].

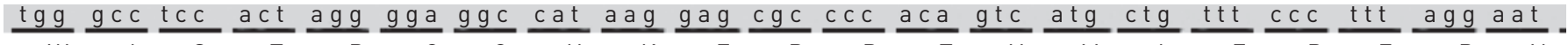

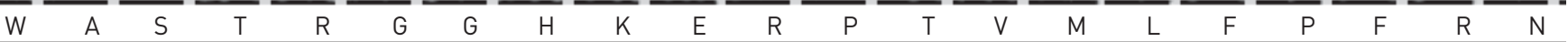
\section{IIIIIIIIIIIIIIIIIIIIIIIIIIIIIIIIIIIIIIIIIIIIII|

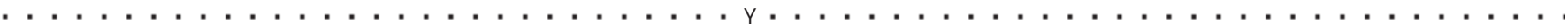

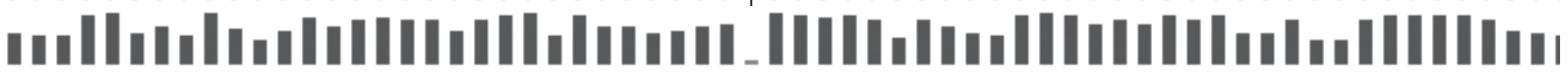
TGGGCCTCCACTAGGGGAGGCCATAAGGAGYGCCCCACAGTCATGCTGTTTCCCTTTAGGAAT

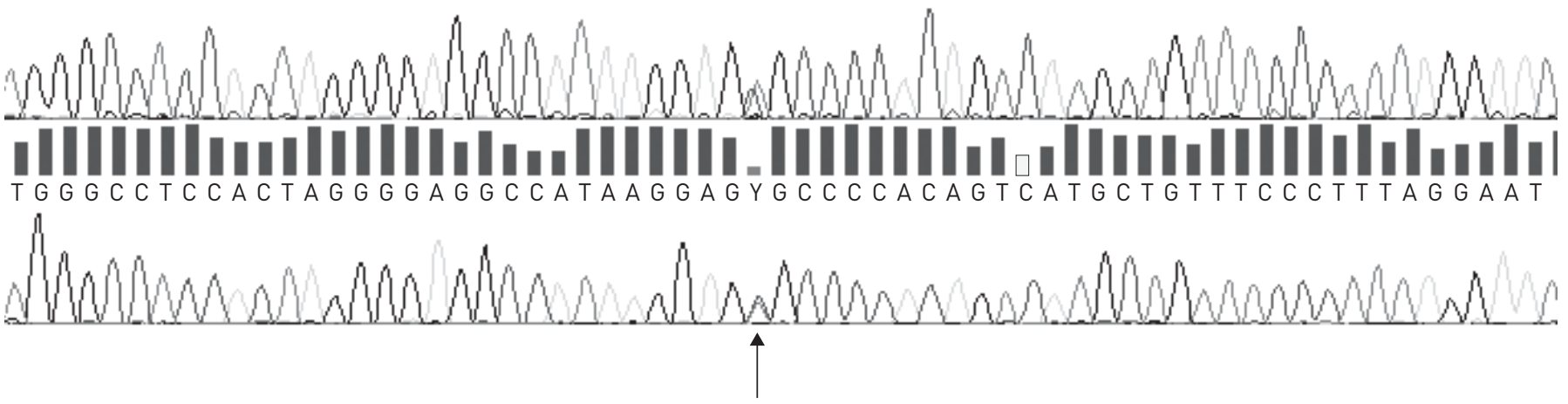

FIGURE 1 Gene mutation analysis of the TINF2 (telomerase repeat binding factor 1-interacting nuclear factor 2) gene by direct sequencing revealed heterozygous c.814C $>\mathrm{T}$, pArg282Cys (arrow) in a 7-year-old child with severe dyskeratosis congenita. 
To verify this hypothesis, one could sequence the TINF2 gene of the patient with DNA from other tissue, a buccal swab and/or a lung sample, where the mutation would probably appear as "really" heterozygous. In addition, high throughput sequencing (with next-generation sequencing) of TINF2 in DNA from the patient's blood should provide precise data on the mutation frequency. However, one cannot definitely exclude the fact that TINF2 mutations could lead to far more heterogeneous clinical phenotypes than previously described. http://ow.ly/vcOaW

Caroline Kannengiesser ${ }^{1}$, Raphael Borie ${ }^{2,3}$ and Patrick Revy ${ }^{4,5}$

${ }^{1}$ Assistance Publique des Hôpitaux de Paris, Hôpital Bichat, Service de Génétique, Université Paris Diderot, Paris, France. ${ }^{2}$ Assistance Publique Hôpitaux de Paris, Hôpital Bichat, Service de Pneumologie A, Centre de Compétence Maladies Rares Pulmonaires, DHU FIRE, Paris, France. ${ }^{3}$ INSERM, Unité 1152, Faculté Bichat, Université Paris 7, Paris, France. ${ }^{4}$ INSERM UMR 1163, Laboratory of Genome Dynamics in the Immune System, Université Paris Descartes, Paris, France.

${ }^{5}$ Sorbonne Paris Cité University, Imagine Institute, Paris, France.

Correspondence: Raphael Borie, Hopital Bichat, Service de Pneumologie A, 46 rue Henri Huchard, Paris 75877 Cedex 18, France. E-mail: raphael.borie@bch.aphp.fr

Received: Feb 262014 | Accepted: Feb 272014

Conflict of interest: None declared.

\section{References}

1 Fukuhara A, Tanino Y, Ishii T, et al. Pulmonary fibrosis in dyskeratosis congenita with TINF2 gene mutation. Eur Respir J 2013; 42: 1757-1759.

2 Armanios MY, Chen JJ, Cogan JD, et al. Telomerase mutations in families with idiopathic pulmonary fibrosis. N Engl J Med 2007; 356: 1317-1326.

3 van Moorsel CH, van Oosterhout MF, Barlo NP, et al. Surfactant protein C mutations are the basis of a significant portion of adult familial pulmonary fibrosis in a Dutch cohort. Am J Respir Crit Care Med 2010; 182: 1419-1425.

4 Walne AJ, Vulliamy T, Beswick R, et al. TINF2 mutations result in very short telomeres: analysis of a large cohort of patients with dyskeratosis congenita and related bone marrow failure syndromes. Blood 2008; 112: 3594-3600.

5 Yamaguchi H, Inokuchi K, Takeuchi J, et al. Identification of TINF2 gene mutations in adult Japanese patients with acquired bone marrow failure syndromes. Br J Haematol 2010; 150: 725-727.

6 Jongmans MC, Verwiel ET, Heijdra Y, et al. Revertant somatic mosaicism by mitotic recombination in dyskeratosis congenita. Am J Hum Genet 2012; 90: 426-433.

Eur Respir J 2014; 44: 269-270 | DOI: 10.1183/09031936.00038714 | Copyright @ERS 2014

\section{From the authors:}

We would like to thank C. Kannengiesser and co-workers for their interest in our article [1] and for their patient's information and comments. They showed that their patient with the TINF2 (telomerase repeat binding factor 1-interacting nuclear factor 2) mutation had the heterozygous mutation, which is usually seen in young patients with dyskeratosis congenita. In addition, they suggested that a somatic reversion might have occurred in our case because figure $1 \mathrm{~b}$ showed that the deletion was not heterozygous despite the late disease onset [1].

Somatic reversion is a possible mechanism, which may explain late disease onset of dyskeratosis congenita with the TINF2 mutation. In fact, JonGMANs et al. [2] described dyskeratosis congenita in a patient with somatic reversion. In this patient, the wild-type allele was observed more than the mutated allele in DNA from his peripheral blood cells despite DNA from his lung tissues revealing heterozygous mutation.

In our case, figure $1 \mathrm{~b}$ showed n871-874 tetranucleotide AGGA deletion in TINF2 gene [1]. However, because the mutation was a deletion mutation, TA cloning was performed for this analysis as described previously [3]. The result of gene mutation analysis by direct sequencing before TA cloning showed that the mutation was heterozygous (fig. 1). Although it may be possible that somatic reversion of the cells in the lungs led to pulmonary fibrosis in our patient, unfortunately we could not analyse DNA from lung tissues because the patient had passed away and no lung tissue had been retained.

More cases are needed to determine the exact mechanism(s) of dyskeratosis congenita by analysing each affected organ/system in detail. 